\title{
Chronic hepatitis C virus (HCV) burden in Rhode Island: modelling treatment scale-up and elimination
}

\author{
A. I. SOIPE ${ }^{1}$, H. RAZAVI ${ }^{2}$, D. RAZAVI-SHEARER ${ }^{2}$, O. GALÁRRAGA ${ }^{3}$, \\ L. E. TAYLOR ${ }^{4}$ AND B. D. L. MARSHALL ${ }^{1 *}$ \\ ${ }^{1}$ Department of Epidemiology, Brown University School of Public Health, Providence, RI, USA \\ ${ }^{2}$ Center for Disease Analysis, Lafayette, CO, USA \\ ${ }^{3}$ Department of Health Services Policy and Practice, Brown University School of Public Health, Providence, RI, \\ USA \\ ${ }^{4}$ Division of Infectious Diseases, The Warren Alpert Medical School of Brown University, and The Miriam \\ Hospital, Center for AIDS Research (CFAR) Providence, RI, USA
}

Received 18 January 2016; Final revision 5 July 2016; Accepted 13 July 2016; first published online 5 August 2016

\section{SUMMARY}

We utilized a disease progression model to predict the number of viraemic infections, cirrhotic cases, and liver-related deaths in the state of Rhode Island (RI) under four treatment scenarios: (1) current HCV treatment paradigm (about 215 patients treated annually, Medicaid reimbursement criteria fibrosis stage $\geqslant F 3$ ); (2) immediate scale-up of treatment (to 430 annually) and less restrictive Medicaid reimbursement criteria (fibrosis stage $\geqslant F 2$ ); (3) immediate treatment scale-up and no fibrosis stage-specific Medicaid reimbursement criteria ( $\geqslant$ F0); (4) an 'elimination' scenario (i.e. a continued treatment scale-up needed to achieve $>90 \%$ reduction in viraemic cases by 2030). Under current treatment models, the number of cirrhotic cases and liver-related deaths will plateau and peak by 2030 , respectively. Treatment scale-up with $\geqslant$ F2 and $\geqslant$ F0 fibrosis stage treatment criteria could reduce the number of cirrhotic cases by $21.7 \%$ and $10 \cdot 0 \%$, and the number of liver-related deaths by $19 \cdot 3 \%$ and $7 \cdot 4 \%$, respectively by 2030 . To achieve a $>90 \%$ reduction in viraemic cases by 2030 , over 2000 persons will need to be treated annually by 2020 . This strategy could reduce cirrhosis cases and liver-related deaths by $78.9 \%$ and $72 \cdot 4 \%$, respectively by 2030 . Increased HCV treatment uptake is needed to substantially reduce the burden of HCV by 2030 in Rhode Island.

Key words: Disease burden, epidemiology, HCV, hepatitis C, modelling, treatment.

\section{INTRODUCTION}

Chronic hepatitis $\mathrm{C}$ virus (HCV) infection is a rapidly growing public health problem in the United States. The consequences of chronic HCV infection are numerous, and include cirrhosis of the liver, liver cancer,

\footnotetext{
* Author for correspondence: B. D. L. Marshall, PhD, Manning Assistant Professor of Epidemiology, Department of Epidemiology, Brown University School of Public Health, 121 South Main Street, Box G-S-121-2, Providence, RI, 02912, USA.

(Email: brandon_marshall@brown.edu)
}

and death [1]. Over the past two decades, morbidity and mortality attributable to HCV infection has continued to increase in parallel with the ageing population (particularly people born between 1945 and 1965 , popularly described as the 'baby boomer' generation) [2]. In the non-institutionalized civilian population in the United States, an estimated 2.7 million people $(\sim 1 \%)$ are chronically infected with HCV [1]. Since 2006, mortality attributable to HCV infection has surpassed that of HIV/AIDS, and chronic liver disease (often HCV-related) is a leading cause of 
death [3]. More people in the United States are now dying of $\mathrm{HCV}$ than all other top 60 nationally notifiable infectious diseases combined [4]. Likewise, the number of $\mathrm{HCV}$-associated advanced liver diseases has been on the rise in the United States and the trend is projected to continue $[1,5]$.

In the state of Rhode Island, it is estimated that between $1.2 \%$ and $1.7 \%$ of the adult population is chronically infected with $\mathrm{HCV}$ [6]. In addition to baby boomers (who account for the majority of people chronically living with HCV infection) younger populations, comprised mainly of people who inject drugs, account for an increasing number of incident $\mathrm{HCV}$ cases in New England [7, 8].

$\mathrm{HCV}$ treatment has evolved rapidly over recent years, particularly with the development of direct-acting antivirals [7]. However, economic and health-systems barriers have greatly limited uptake of more effective, safer therapies [9]. Under the current HCV treatment paradigm, the burden of $\mathrm{HCV}$ infection and its sequelae are expected to continue on an upward trend in the United States and other industrialized countries, particularly as the number of people with advanced HCV-related liver disease grows [10, 11]. Moreover, many current Medicaid reimbursement criteria (with various liver staging, substance use-related, and other restrictions) do not follow recommendations from professional organizations, such as the Infectious Diseases Society of America and the American Association for the Study of Liver Diseases [12]. Therefore, in order to ensure uptake of novel anti-HCV drugs, greatly decreased prices of therapy, and a change in HCV treatment policies, are needed.

In response to the high morbidity and mortality associated with HCV infection, we launched Rhode Island Defeats Hepatitis C ('RI Defeats Hep C'), a project dedicated to the elimination of $\mathrm{HCV}$ in Rhode Island. A key objective of RI Defeats Hep C is to identify the most effective HCV treatment and prevention policies that will lead to a substantial decrease, and eventual elimination, of chronic HCV infection in Rhode Island. To meet this objective, we carried out a mathematical modelling analysis of different treatment scenarios, using as a base case $\mathrm{HCV}$ treatment roll-out under the current Rhode Island Medicaid HCV treatment policies [12, 13].

\section{METHODS}

To estimate the burden of chronic HCV infection in Rhode Island, we adapted an $\mathrm{HCV}$ disease progression model, described in detail previously [5, 14]. Wherever possible, state-specific estimates were used to construct a model representing the adult population of Rhode Island. The model was designed to examine the effects of possible policy changes and interventions on the burden of $\mathrm{HCV}$ in the state. The aim of this study was to estimate the projected burden of the disease under different treatment scenarios, and also to demonstrate whether HCV disease burden can be reduced via change in relevant treatment scale-up and eligibility policies.

In brief, a computer-generated simulation model of $\mathrm{HCV}$ burden was used to investigate four possible treatment scenarios. The model was constructed in Microsoft Excel (Microsoft Corp., USA), and Monte Carlo and sensitivity analyses were done using Crystal Ball, an Excel add-in by Oracle. We used beta-Project Evaluation and Review Technique (PERT) distributions to model the uncertainty associated with the inputs. Tracking of the HCV-infected population started from 1950, and was calibrated to 2014; the model projected the outcomes up to 2030. The number of individuals infected with HCV prior to 1950, who are still alive, was expected to be negligible and to have minimal impact on the final analysis. The model population was tracked by 5 -year age cohorts and by gender. To simulate ageing, each year one-fifth of the age group moved to the next age cohort, after accounting for agespecific mortality (see below). The distribution of HCV prevalence for the age and gender cohorts was assumed to be proportional to the US population [1], given that the age and gender structures in Rhode Island are very similar to the US average [6]. Incident cases were distributed in the different age and gender cohorts using distributions reported by the Centers for Disease Control and Prevention (CDC) between 1992 and 2007 [15-18]. We assumed that the incidence distribution from 2008 to 2030 remained stable and reflected that reported in 2007.

The disease progression modelling was carried out via multiplying the total number of cases at a particular stage of the disease by a progression rate to the next stage (see disease progression schematic, Supplementary Fig. S1). Subjects in a particular disease stage were handled as stocks, while yearly transitions from one health state to another were treated as flows. HCV disease progression rates are presented in Supplementary Tables S1 and S2), and were adapted from previously published studies [19-22] or back-calculated. As described previously [14], the number of reported cases of liver cancer and liver cancer deaths (by age and 
gender) from the US Surveillance, Epidemiology, and End Results (SEER) database were used to backcalculate age- and gender-specific annual fibrosis progression rates (Supplementary Table S1). The transition rates shown in Supplementary Table S1 equate to $\sim 16 \%$ cirrhotic at 20 years, which is within the range of empirical estimates [23], and similar to that of a recently published modelling study that estimated a $12-14 \% \mathrm{cu}-$ mulative cirrhosis progression rate at 20 years [24]. For persons acutely infected, we estimated the proportion that progressed to chronic infection, taking into account the spontaneous clearance of the virus based on previously published data [6, 25] (see Supplementary Table S2).

The model estimated the annual number of acute infections historically (from 1950) using a calibration procedure described previously [5]. In brief, national surveillance data [26] regarding the annual number of estimated total new infections was adjusted for the state's population relative to the US population in each year. These values reflect methods used by the $\mathrm{CDC}$ to estimate true incidence of acute $\mathrm{HCV}$ infection, given under-ascertainment of reported cases [27]. For 2010 onwards, we used national surveillance data and other previously published estimates [14, 28-30], and interpolated the number of acute infections in Rhode Island, assuming constant incidence over the simulation period. The prevalence of chronic HCV infection in a particular year was calculated by the sum of new infections (incidence) minus disease stage-specific mortality and cured cases leading up to that year. Finally, incidence was modified to match reported prevalence in 2014 in Rhode Island (see below). The final incidence values for each year, as well as the interpolated CDC surveillance estimates for Rhode Island, are shown in Supplementary Fig. S2).

To parameterize the model, data on Rhode Island-specific parameter estimates were abstracted from published reports and from other data sources $[3,6,14]$. The following parameters were quantified using state-specific data and are summarized in Table 1: the size of the HCV-infected population (both anti-HCV positive and viraemic infections), proportion medically eligible for $\mathrm{HCV}$ treatment, the state's HCV genotype distribution, and the number liver transplants annually. For example, chronic HCV prevalence was obtained from a Rhode Island study [6] that used a method similar to that employed in a recent analysis of national HCV prevalence [31]. In both studies, the $\mathrm{HCV}$ prevalence estimate from the National Health and Nutrition Examination
Survey (NHANES) was supplemented with data from grey literature and other sources to account for missed cases from under-represented populations and/or those purposely excluded from the NHANES (e.g. veterans, the homeless). The chronic HCV prevalence estimate obtained by Kinnard et al. was also adjusted to account for the fact that the racial composition of Rhode Island differs from that of the national average [6].

The following parameters were based on national data and interpolated to the Rhode Island population ( $0.33 \%$ of the US population in 2014$)$ : proportion previously diagnosed, number newly diagnosed per year, proportion of the diagnosed pool in each fibrosis liver disease stage, and the number of treated and cured patients per year [3, 20, 32-36]. For example, the annual number of treated and cured patients in Rhode Island between 2004 and 2007 was estimated from previously published national data [36], assuming a sustained virological response (SVR) of 55\% for G1, $70 \%$ for $\mathrm{G} 2$ and $\mathrm{G} 3$, and $48 \%$ for G4, and a treatment completion rate of $80 \%$ [5]. The number of cured patients prior to 2002 was ignored and assumed not to have an effect on the primary results. The number of cured patients between 2008 and 2013 was extrapolated based on results of a previously published modelling study [36]. Only patients with fibrosis $\geqslant F 2$ and aged between 15 and 64 years were considered for treatment prior to 2014 .

Background mortality rates by age and gender were adjusted for increased mortality in $\mathrm{HCV}$-infected patients due to injection drug use and transfusion, as described in detail previously [37]. These rates were applied to all HCV-infected populations in the model. Separate mortality rates were defined for liver-related deaths in individuals with decompensated cirrhosis (diuretic sensitive and refractory ascites, variceal haemorrhage, and hepatic encephalopathy), hepatocellular carcinoma (HCC), and those who required liver transplantation (sources and rates shown in Supplementary Table S2).

Following model calibration, we probed the model with four treatment scenarios, resulting in future projections of HCV disease burden. The base-case scenario describes the extant $\mathrm{HCV}$ treatment paradigm, in which about 215 patients are treated annually, with a restriction to patients in fibrosis stage $\geqslant \mathrm{F} 3$ (based on current Rhode Island Medicaid reimbursement criteria) [12]. Second, we analysed two treatment scale-up scenarios. The first describes an immediate scale-up of treatment to 430 patients annually, and a 
Table 1. Summary parameters for Rhode Island HCV burden model, base case (2014)

\begin{tabular}{lllll}
\hline \hline Variable & Base estimate & Low & High & Source \\
\hline HCV Ab+ positive (prevalence) & $19632(2 \cdot 0 \%)$ & $16603(1 \cdot 7 \%)$ & $22660(2 \cdot 3 \%)$ & {$[6]$} \\
Chronic infections (prevalence) & $14527(1 \cdot 5 \%)$ & $12286(1 \cdot 2 \%)$ & $16768(1 \cdot 7 \%)$ & {$[6]$} \\
Annual number of new infections & 86 & - & 400 & {$[14,28-30]$} \\
Spontaneous clearance rate & $18 \%$ & $15 \%$ & $45 \%$ & {$[25]$} \\
Previously diagnosed & $49 \%$ & - & $75 \%$ & {$[32,33]$} \\
Newly diagnosed (number per year) & 360 & 65 & 654 & {$[3]$} \\
Proportion of diagnosed pool in F0 & $15 \%$ & $13 \%$ & $17 \%$ & {$[20,34]$} \\
Proportion medically eligible for treatment & $95 \%$ & - & - & L. Taylor, pers. comm. \\
Age range for treatment & $18-70$ & - & - & L. Taylor, pers. comm. \\
Treatment restriction & $\geqslant F 3$ & - & - & {$[45]$} \\
Treated patients (number per year)* & 215 & - & 322 & {$[5,36]$} \\
Genotype distribution & & & & {$[38]$} \\
G1a & $46 \cdot 2 \%$ & - & - & \\
G1b & $26 \cdot 3 \%$ & - & - & \\
G2 & $10 \cdot 7 \%$ & - & - & \\
G3 & $8 \cdot 9 \%$ & - & - & \\
G4 & $6 \cdot 3 \%$ & - & - & \\
Other & $1 \cdot 6 \%$ & - & - & \\
Average SVR* & & - & - & \\
G1a & $90 \%$ & - & - & \\
G1b & $90 \%$ & - & - & \\
G2 & $90 \%$ & - & - & \\
G3 & $75 \%$ & - & - & \\
G4 & $90 \%$ & - & - & \\
Other & $90 \%$ & - & - & \\
Number of liver transplants (annually) & 10 & - & - & \\
\hline \hline
\end{tabular}

HCV, Hepatitis C virus; SVR, sustained virological response; pers. comm., personal communication.

* Average SVR increases to $90 \%$ for all genotypes in 2015 .

less restrictive Medicaid treatment authorization and reimbursement criteria (fibrosis stage $\geqslant \mathrm{F} 2$ ); the other treatment scale-up scenario describes an immediate treatment scale-up to 430 patients annually and no fibrosis stage-specific Medicaid reimbursement criteria $(\geqslant F 0)$. Finally, there is an elimination scenario, in which the number of patients treated annually is determined based on a continued treatment scale-up needed to achieve $>90 \%$ reduction in viraemic cases by 2030 . In the elimination scenario, there is no treatment criteria based on liver fibrosis stage. In both scenarios in which there is no fibrosis-stage criteria, treatment is independent of an individual's disease stage (i.e. treatment is allocated randomly throughout the population).

The number of persons in each liver fibrosis stage (including the number of cirrhotic patients), and the number of deaths from liver-related and all causes in the $\mathrm{HCV}$-infected population was estimated for the base case and each treatment scenario, using the HCV disease progression framework described above and previously $[6,14]$. The average SVR rates for each genotype (assuming direct-acting antiviral therapy) were based on previously published data [39], and are shown in Table 1.

Finally, we carried out a sensitivity analysis and estimated the $95 \%$ uncertainty interval for chronic $\mathrm{HCV}$ prevalence, number of cirrhotic cases, as well as overall and liver-related mortality in the base-case scenario. Monte Carlo simulations were conducted to examine the effect of variability in estimated $\mathrm{HCV}$ incidence and other factors. We conducted Monte Carlo analyses in which input parameters were randomly sampled from beta-PERT distributions. Specifically, for each model input that was considered as having uncertainty, a beta-PERT distribution was developed with a minimum and maximum value defined by the low and high ranges shown in Table 1 and Supplementary Table S2, and a likeliest value given by that used in the primary base-case analysis. As an example a beta-PERT distribution is shown in Supplementary Figure S3. As shown, we focused our sensitivity analysis on parameters for which there was greater uncertainty (e.g. HCV incidence, 


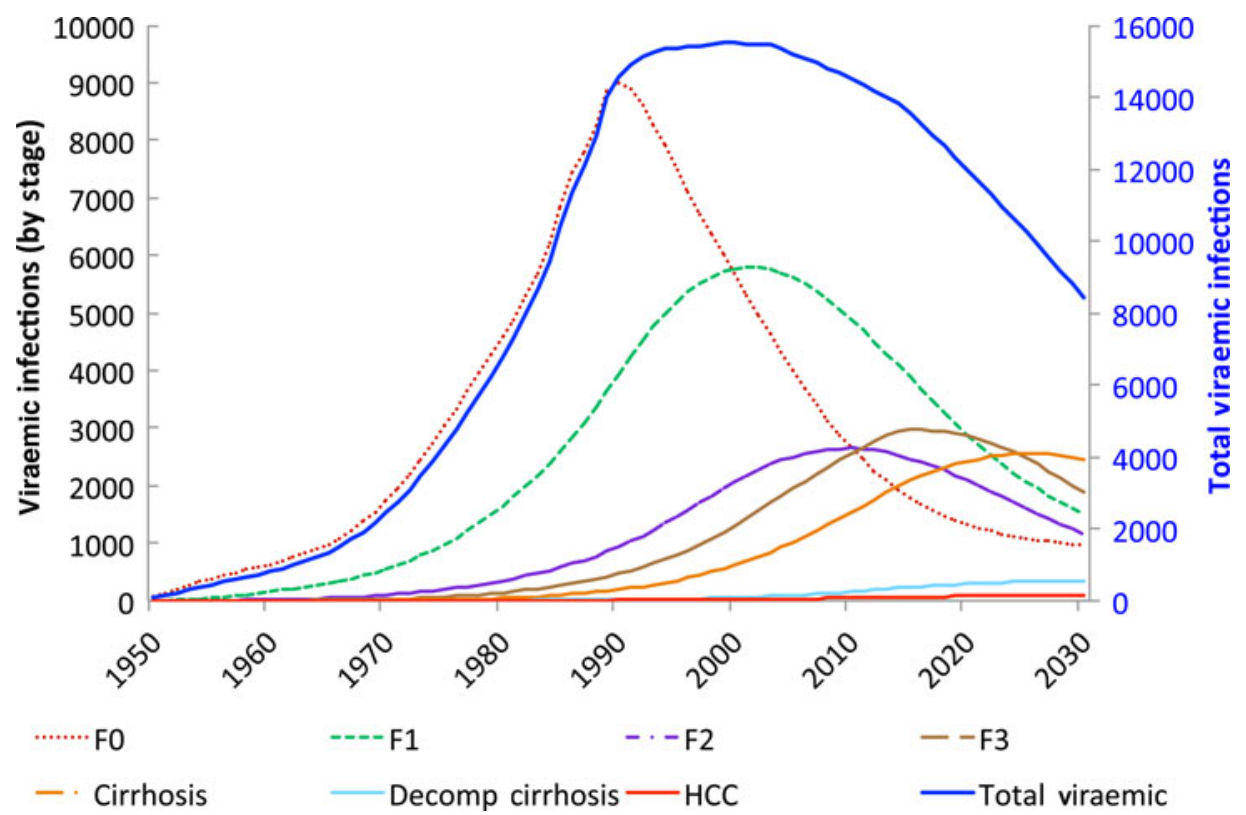

Fig. 1. Estimated burden of chronic HCV infection in Rhode Island, 1950-2030: total viraemic infections (dark blue) and by liver disease stage. HCC, Hepatocellular carcinoma.

number newly diagnosed per year, liver-related deaths, etc.) Finally, to further examine the impact of uncertainty regarding specific variables (e.g. disease progression rates), we conducted a series of one-way sensitivity analyses, using the same methodology described above but sampling from only one beta-PERT distribution for each analysis.

\section{RESULTS}

The peak prevalence of chronic $\mathrm{HCV}$ infection in Rhode Island was estimated to occur in 1999, with 15500 viraemic cases (Fig. 1). The estimated number of new infections per year (which was estimated to peak in 1989) is shown in Supplementary Figure S1. The number of HCV-infected individuals with fibrosis stage F0 has been declining since 1990, while those in fibrosis stage F2 likely peaked in 2010 (Fig. 1). Assuming the current treatment paradigm continues, this number is expected to drop below 10000 viraemic cases by the year 2030. However, the number of $\mathrm{HCV}$-infected persons with decompensated cirrhosis and HCC in the Rhode Island population is expected to continue to rise as the baby-boomer generation ages (see Fig. 1). In the base-case scenario, the proportion of the diagnosed pool in F0 is estimated to increase from $15 \%$ in 2014 to $90 \%$ by 2025 .

The annual number of patients treated in the base case and various treatment scale-up scenarios is shown in Figure 2. In the elimination scenario, the number of patients treated was back-calculated to result in $<1000$ viraemic cases (i.e. a $\geqslant 90 \%$ reduction) by 2030 . As shown, achieving elimination by 2030 requires a rapid and substantial scale-up in treatment, to over 2000 patients treated annually by 2020 .

The estimated total number of viraemic infections and cirrhotic cases under each scenario is shown in Figures 3 and 4, respectively. Immediate treatment scale-up with $\geqslant \mathrm{F} 2$ and $\geqslant \mathrm{F} 0$ fibrosis stage treatment criteria could reduce the total number of viraemic cases in 2030 by $17 \cdot 3 \%$ and $19 \cdot 6 \%$, respectively (Fig. 3). In contrast, a greater reduction in cirrhotic cases was observed when treatment was restricted to persons in liver disease stage $\geqslant \mathrm{F} 2(21 \cdot 7 \%$ vs. $10 \cdot 0 \%$ reduction for the $\geqslant \mathrm{F} 0$ scenario in 2030 , respectively). As expected, the largest reduction in total cases and cirrhotic patients was observed in the elimination scenario $(93.9 \%$ and $78.9 \%$, respectively).

Under the present treatment paradigm, the rates of all-cause and liver-related deaths are expected to continue to increase and peak by 2030 (Figs 5 and 6, respectively). Under the elimination scenario (i.e. $\geqslant 90 \%$ reduction in viraemic cases), projected mortality associated with any cause, as well as mortality from liver-related conditions, is expected to fall through to 2030 (Figs 5 and 6). This treatment strategy could reduce liver-related deaths by $72.4 \%$ in 2030 compared to the base scenario. Reductions in 


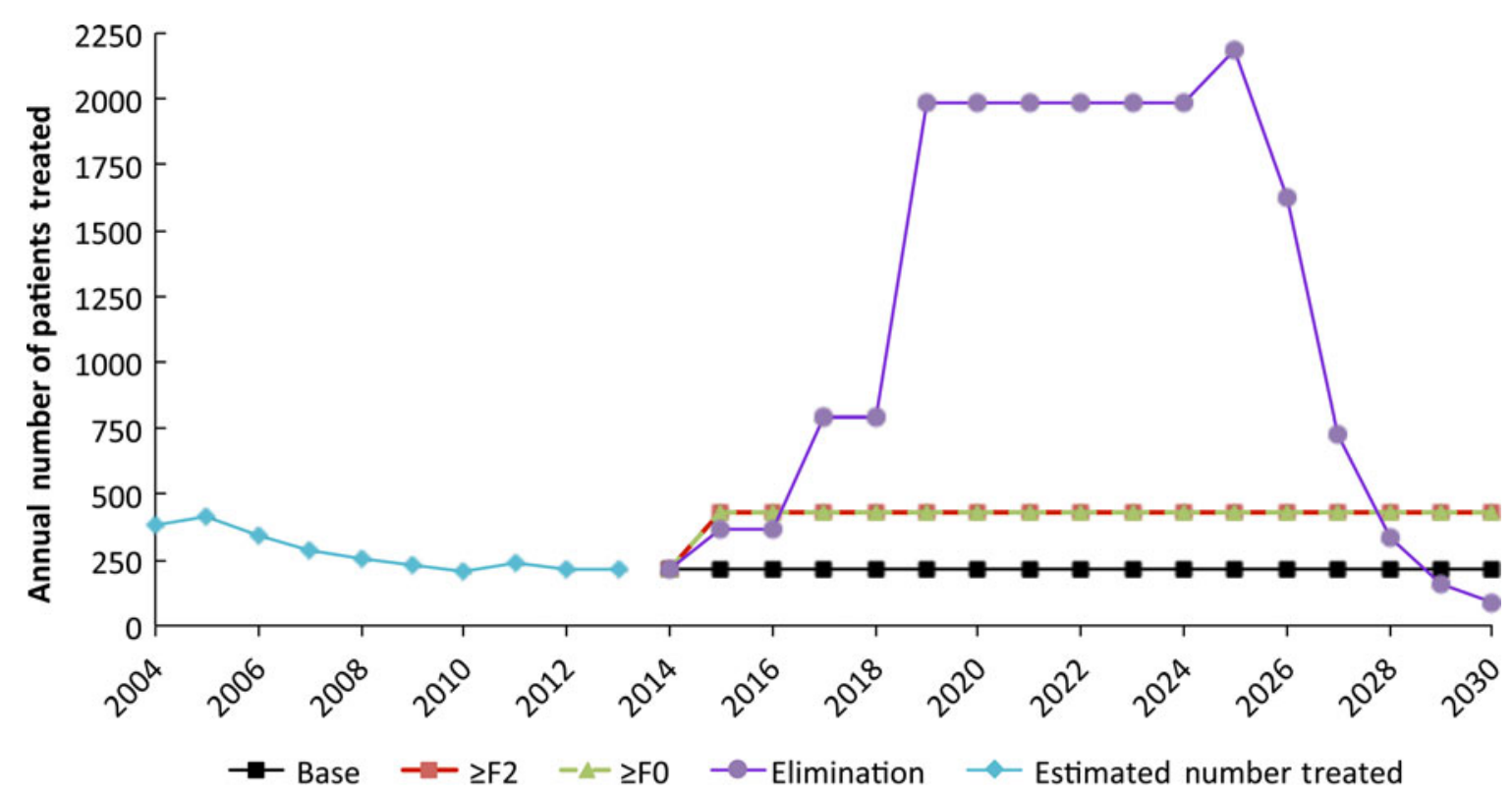

Fig. 2. Annual number of patients treated in the base-case and treatment scale-up scenarios, Rhode Island, $2004-2030$. The annual number of treated patients between 2004 and 2013 was estimated from national data [36], and interpolated to the Rhode Island population.

mortality were also observed in the treatment scale-up scenarios. As shown in Figures 5 and 6, assuming the same number of persons are treated annually, restricting treatment eligibility to $\geqslant \mathrm{F} 2$ produced greater reductions in all-cause and liver-related deaths compared to the $\geqslant \mathrm{F} 0$ scenario.

The results of the one-way sensitivity analyses are summarized in a tornado diagram (see Supplementary Fig. S4). As shown, the model was most sensitive to the number of incident cases. Estimated chronic HCV prevalence in 2030 was also sensitive to changes in disease progression rates, including the rate at which individuals transition from mild to moderate fibrosis.

\section{DISCUSSION}

The results from our mathematical modelling study show that HCV-related morbidity and mortality can be reduced significantly in Rhode Island if an aggressive treatment strategy is implemented over the next decade. In contrast, if the current rate of HCV treatment continues, the number of liver-related deaths will continue to increase until at least 2030. A treatment scenario in which the number of patients treated annually is increased to 430 reduces the burden of advanced liver disease and related deaths in Rhode Island, although the estimates fall within the uncertainty interval of the base-case scenario. However, our model suggests that HCV elimination (i.e. $<1000$ chronic HCV cases by 2030) is the best approach and notably falls outside the uncertainty interval. Reducing viraemic cases by $90 \%$ is possible if treatment is scaled up immediately and continuously, to 2000 persons annually, by 2020 .

Our results are in accordance with a recently published national study that found a marked scale-up of treatment is needed to reduce future HCV disease burden [39]. In that study, it was projected that treating all diagnosed patients in the United States would reduce $\mathrm{HCV}$-related disease burden to $<1400$ cases in 50 years, although such a strategy would be extraordinarily expensive. The authors also found that treating $5 \%$ of all diagnosed patients annually, irrespective of disease stage, would produce substantial public health benefit and would be much more affordable. These findings are similar to our results, in that substantial reductions in chronic HCV prevalence will likely require immediate and ongoing treatment of a significant proportion of the population over the next decade.

A comparison of model outputs from scenarios in which treatment is capped (but restricted to individuals with liver disease stage $\geqslant F 2$ and $\geqslant F 0$ ), shows that, although the estimated number of viraemic infections in 2030 was similar, a greater reduction in cirrhotic cases was observed when treatment was restricted to persons in liver disease stage $\geqslant \mathrm{F} 2$. More advanced $\mathrm{HCV}$ disease stages have a higher 


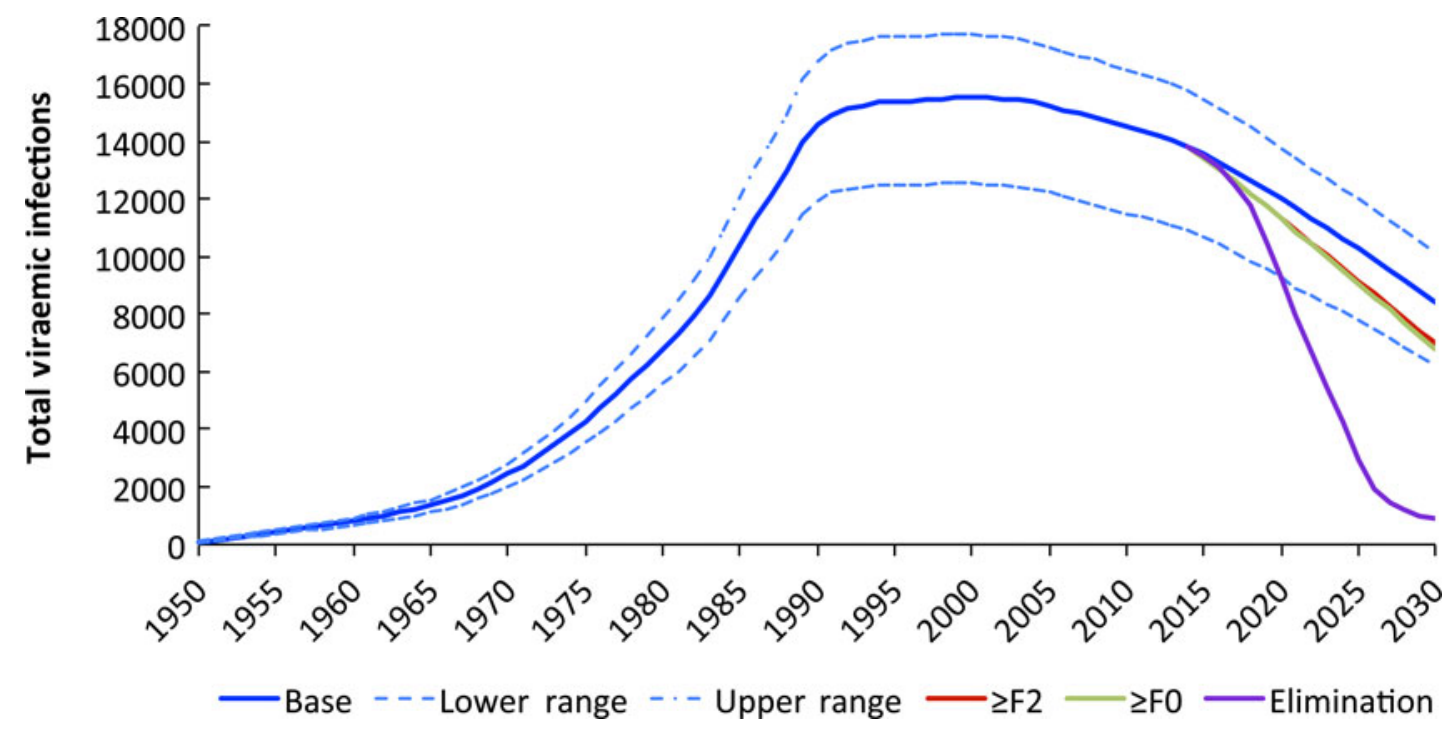

Fig. 3. Estimated number of total viraemic infections in Rhode Island, 1950-2030: base-case and treatment scale-up scenarios. Results of the Monte Carlo sensitivity analysis (95\% uncertainty interval, base case) are shown by dashed blue lines. The $95 \%$ uncertainty interval at 2030 is $6260-10490$ total viraemic infections.

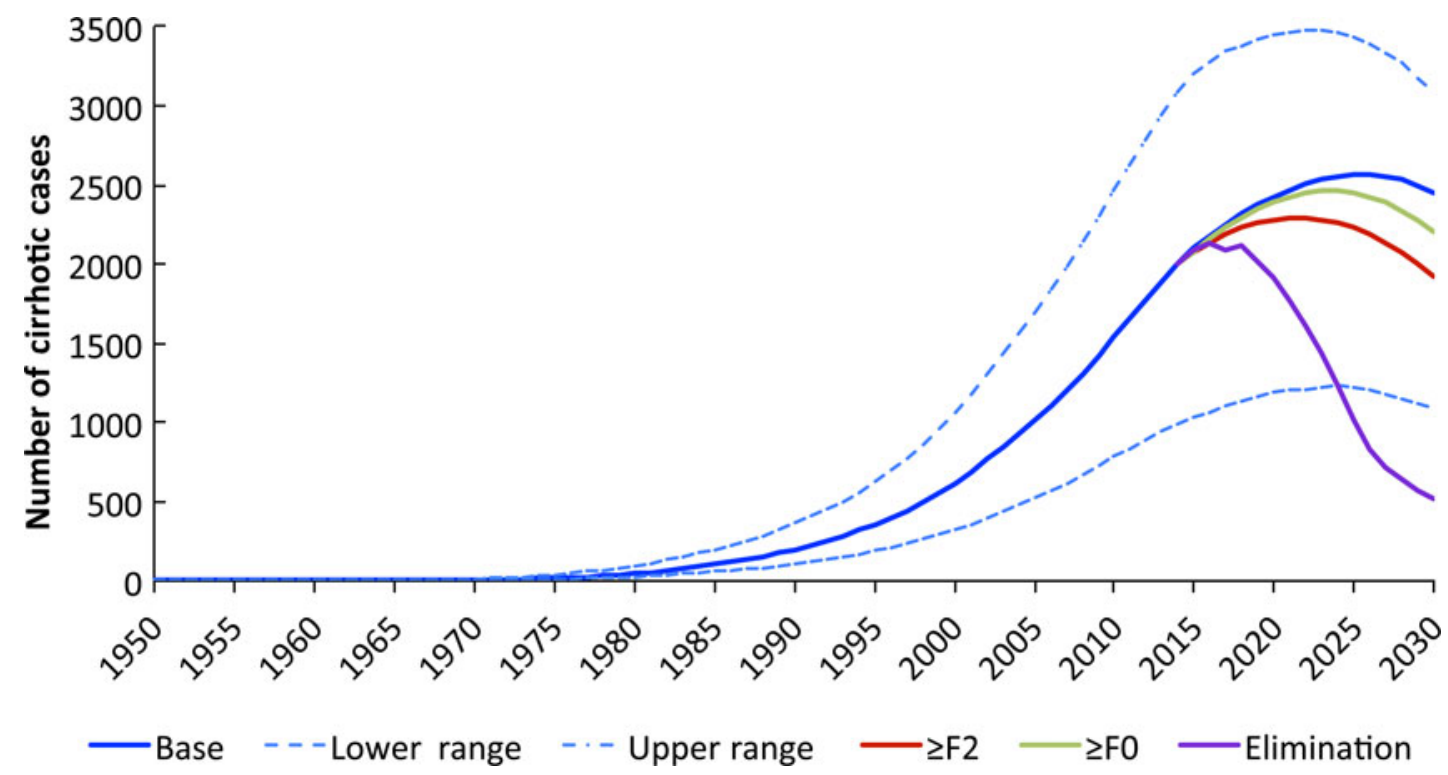

Fig. 4. Estimated number of cirrhotic cases in Rhode Island, 1950-2030: base-case and treatment scale-up scenarios. Results of the Monte Carlo sensitivity analysis (95\% uncertainty interval, base case) are shown by dashed blue lines. The $95 \%$ uncertainty interval at 2030 is $1085-3092$ cirrhotic cases.

probability of progression; as such, the model confirms that restricting treatment to these patients has a larger impact on rates of cirrhosis and liverrelated mortality in the population. However, our results further imply that a significant proportion of persons in lower fibrosis disease stages will eventually progress to F2. This is in agreement with a study demonstrating substantial natural progression in liver fibrosis from stage F0/F1 to higher stages [40]. Thus, if achieving $>90 \%$ reduction in viraemic cases is the goal, a rapid expansion of treatment eligibility to all patients (regardless of disease stage) is recommended.

This study has a number of important limitations. First, in our primary analysis, we assumed a constant incidence of new infections over the modelled time period (2015-2030) in all scenarios. Recent studies 


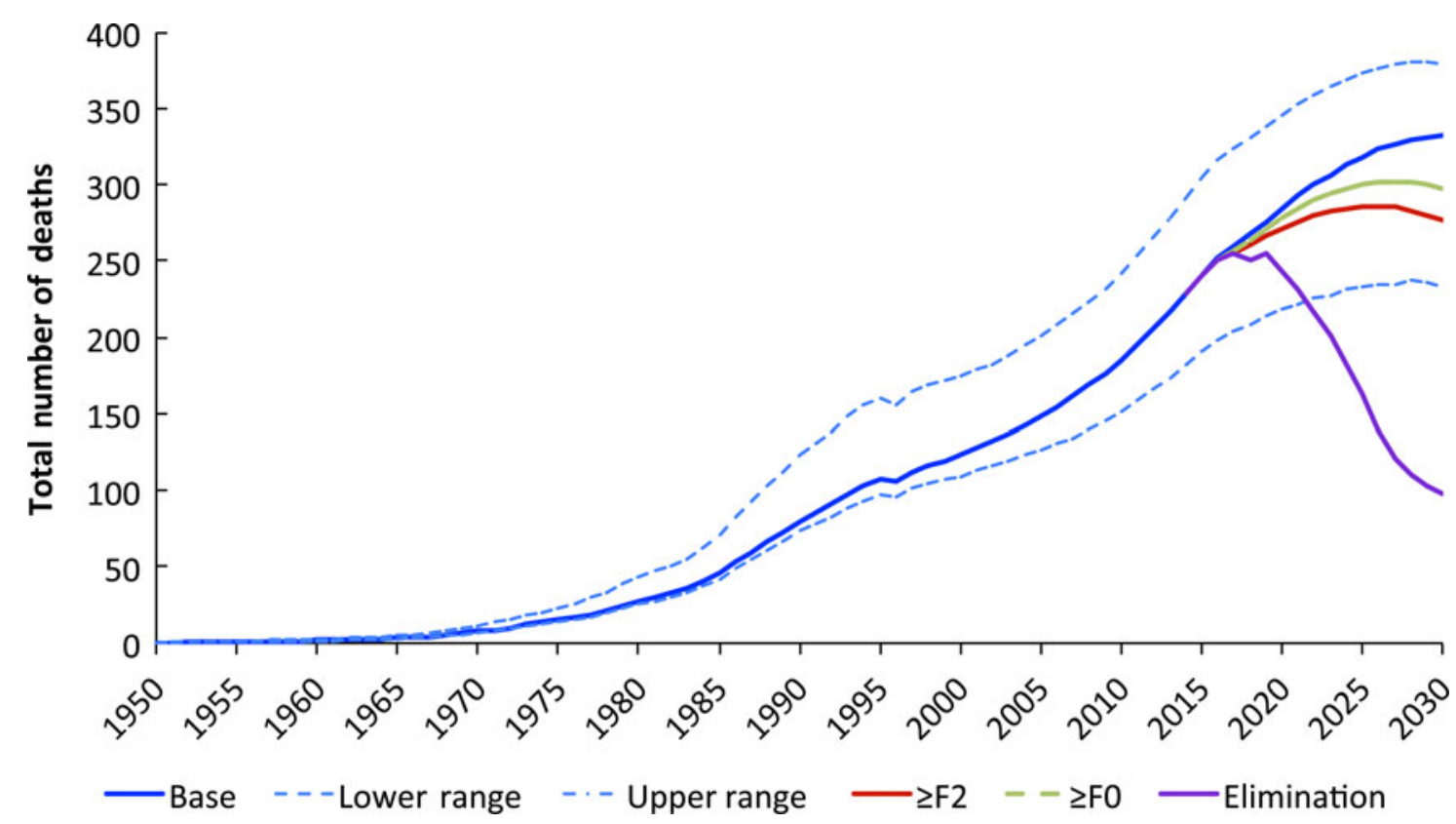

Fig. 5. Estimated number of deaths from any cause in the viraemic HCV population, Rhode Island, 1950-2030: base-case and treatment scale-up scenarios. Results of the Monte Carlo sensitivity analysis $(95 \%$ uncertainty interval, base case) are shown by dashed blue lines. The $95 \%$ uncertainty interval at 2030 is $237-381$ deaths.

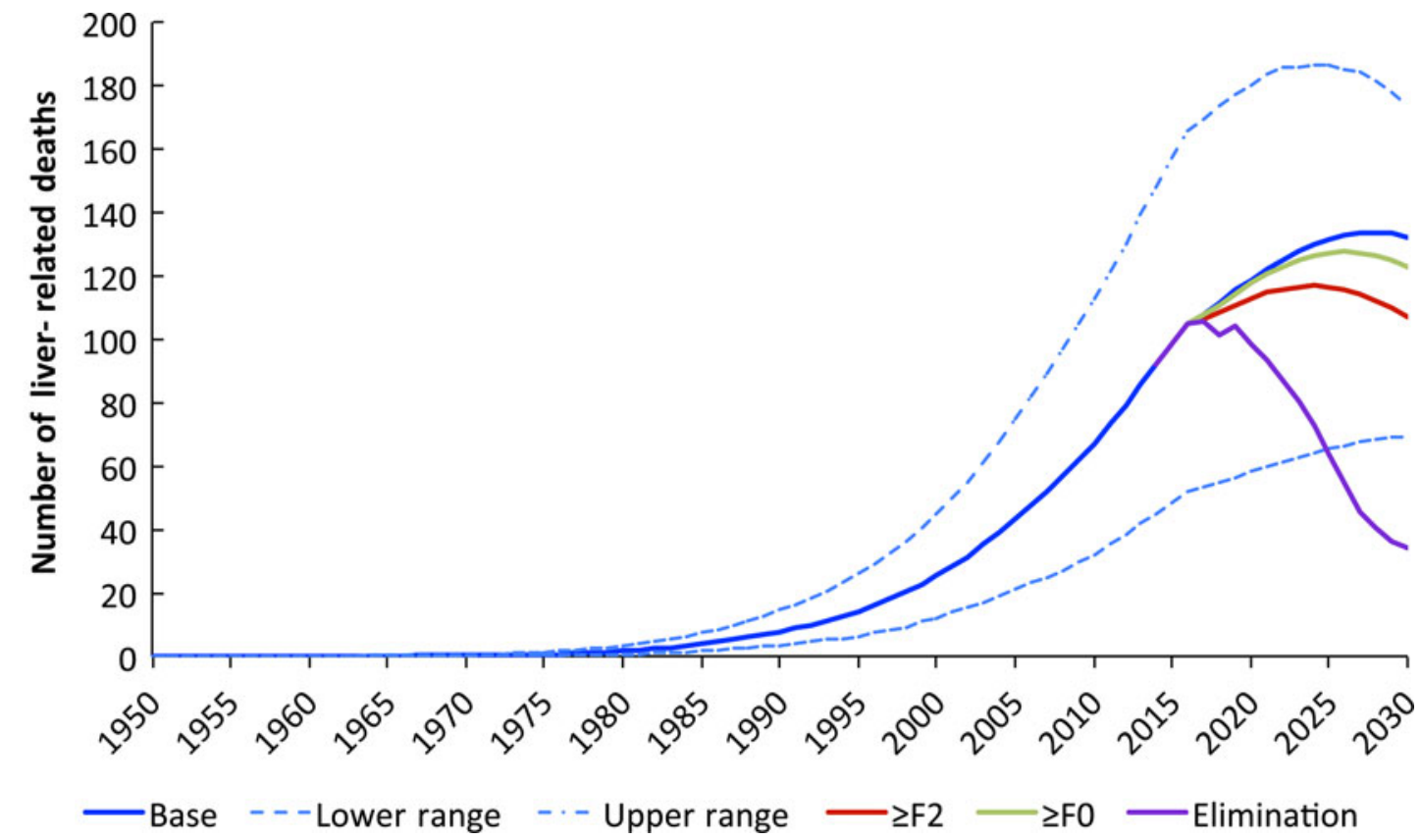

Fig. 6. Estimated number of liver-related deaths in the viraemic HCV population, Rhode Island, 1950-2030: base-case and treatment scale-up scenarios. Results of the Monte Carlo sensitivity analysis $(95 \%$ uncertainty interval, base case) are shown by dashed blue lines. The $95 \%$ uncertainty interval at 2030 is $69-177$ liver-related deaths.

have identified an increasing number of acute $\mathrm{HCV}$ infections in young people who inject drugs in the United States and in New England [8, 41, 42]. Thus, the number of new infections in Rhode Island may be greater than we estimated, and could continue to increase over time. In addition to the removal of restrictions on $\mathrm{HCV}$ treatment eligibility for people who inject drugs [12], the expansion of additional 
prevention and harm reduction interventions, including needle-and-syringe programmes, is recommended. Similarly, we did not consider treatment as prevention (i.e. the impact of curing $\mathrm{HCV}$ in transmitters on $\mathrm{HCV}$ incidence and prevalence).

Second, we were not able to obtain specific estimates for the number of treated patients from Rhode Island Medicaid, private insurance companies, or from the Veterans Affairs system. Therefore, the estimated number of treated patients may be inaccurate.

Third, because mortality rates were modelled based on age, gender, injection drug use, and liver-related deaths without accounting for deaths from other risk factors, our model may underestimate the true HCVrelated mortality. Under-reporting of mortality rates from HCV has been noted in previous studies, where researchers found under-documentation of HCV infection on death certificates [43]. We attempted to account for this uncertainty by conducting sensitivity analyses in which the likelihood of death at each HCV disease stage was varied.

Fourth, our model assumed the same rate of spontaneous clearance for all HCV-infected individuals. Recent work has shown that the rate of spontaneous clearance may be as high as $50 \%$ in persons with reinfection [44]. Future work will seek to incorporate differential rates of spontaneous clearance based on re-infection status and other risk factors.

Fifth, as with all modelling studies, our findings are only as valid as the available data and assumptions. Given that HCV surveillance (including case notification) and the dissemination of information regarding HCV treatment uptake is limited in Rhode Island [45], data exclusively representative of the state's population were frequently unavailable. As such, a limitation of our study is the use of national estimates from which state-specific figures were interpolated, since actual values for the population under study were unavailable in Rhode Island. Wherever possible, modelling parameter estimates were derived from published literature of Rhode Island-specific data [6]. However, we note that national estimates have been validated and utilized successfully in prior modelling studies $[5,14,46]$. This limitation demonstrates the urgent need to improve HCV surveillance capacity and to increase research and treatment evaluation infrastructure in Rhode Island.

Sixth, with the discovery of more potent anti-HCV medications in the future, the SVR rates specified in the input parameters might be different from presentday estimates. This may alter the true progression and prevalence estimates, particularly for later years of the simulation.

Seventh, the model utilized in this analysis is a progression model in which costs of treatment were not estimated. Therefore, we were unable to determine the budgetary implications of the simulated treatment scale-up strategies. Recent research has shown that treating HCV infection at early fibrosis stages is costeffective (at a standard cut-off of $<\$ 10000$ per quality-adjusted life-year gained), but that such strategies incur substantial upfront investment [47]. Future work will investigate the cost-effectiveness of various treatment strategies in Rhode Island.

A final limitation of our study is the assumption that sufficient numbers of patients will be screened and diagnosed in order to achieve the modelled treatment targets: this is particularly an issue under the elimination scenario $(>90 \%$ reduction in viraemic cases). Generally, as treatment rate ramps up, it may become more difficult to find untreated patients. Low-threshold, community-based $\mathrm{HCV}$ screening programmes with reflexive RNA confirmatory testing are needed to ensure a sufficient number of individuals are eligible for treatment.

In sum, our analysis demonstrates that achieving a substantial reduction in the disease burden of HCV in Rhode Island will necessitate treating 2000 patients annually by 2020 . However, there is a need to enhance $\mathrm{HCV}$ surveillance in Rhode Island and to increase clinical infrastructure and provider capacity to meet the demands of treatment scale-up. If the current paradigm of treating a much smaller number of patients with F2 liver staging and above is sustained, it will take a much longer period to eliminate $\mathrm{HCV}$ infection in Rhode Island, with total mortality increasing until at least 2030.

\section{SUPPLEMENTARY MATERIAL}

For supplementary material accompanying this paper visit http://dx.doi.org/10.1017/S0950268816001722.

\section{ACKNOWLEDGEMENTS}

The authors acknowledge support from Rhode Island Defeats HepC through a Rhode Island Innovation Fellowship to Lynn E. Taylor, MD from the Rhode Island Foundation. The authors also acknowledge in-kind support provided by the Center for Disease Analysis. Brandon Marshall is supported by a Henry Merrit Wriston Fellowship from Brown 
University and by the National Institute on Drug Abuse (DP2-DA040236). The decision to write-up this manuscript for publication and the contents are the sole responsibilities of the authors.

\section{DECLARATION OF INTEREST}

The Center for Disease Analysis has received research funding from public and private sources (Gilead Sciences, Boehringer Ingelheim and AbbVie), but its projects are limited to basic epidemiology and modelling research. H. Razavi and D. Razavi-Shearer are employees of The Center for Disease Analysis but did not receive remuneration for this work. All other authors declare no conflicts of interest.

\section{REFERENCES}

1. Denniston MM, et al. Chronic hepatitis $\mathrm{C}$ virus infection in the United States, national health and nutrition examination survey 2003 to 2010. Annals of Internal Medicine 2014; 160: 293-300.

2. Jacobson IM, et al. Prevalence and challenges of liver diseases in patients with chronic hepatitis $\mathrm{C}$ virus infection. Clinical Gastroenterology and Hepatology 2010; 8: 924-933.

3. Klevens RM, et al. Evolving epidemiology of hepatitis C virus in the United States. Clinical Infectious Diseases 2012; 55: S3-S9.

4. Ly KN, et al. Rising mortality associated with hepatitis $\mathrm{C}$ virus in the United States, 2003-2013. Clinical Infectious Diseases 2016; 62: 1287-1288.

5. Razavi H, et al. Chronic hepatitis $\mathrm{C}$ virus (HCV) disease burden and cost in the United States. Hepatology 2013; 57: 2164-2170.

6. Kinnard EN, et al. Estimating the true prevalence of hepatitis $\mathrm{C}$ in rhode island. Rhode Island Medical Journal 2014; 97: 19-24.

7. Shiffman ML. Hepatitis C virus therapy in the direct acting antiviral era. Current Opinion in Gastroenterology 2014; 30: 217-222.

8. Stein MD, Maksad J, Clarke J. Hepatitis C disease among injection drug users: Knowledge, perceived risk and willingness to receive treatment. Drug and Alcohol Dependence 2001; 61: 211-215.

9. McGowan CE, Fried MW. Barriers to hepatitis C treatment. Liver International 2012; 32: 151-156.

10. Ward JW, Rein DB, Smith BD. Data to guide the 'test and treat era' of hepatitis C. Gastroenterology 2012; 143: 887-889.

11. Kabiri M, et al. The changing burden of hepatitis $C$ virus infection in the United States: Model-based predictions. Annals of Internal Medicine 2014; 161: 170-180.

12. Barua $\mathbf{S}$, et al. Restrictions for medicaid reimbursement of sofosbuvir for the treatment of hepatitis $\mathrm{C}$ virus infection in the United States. Annals of Internal Medicine 2015; 163: 215-223.
13. Nguyen JT, et al. A budget impact analysis of newly available hepatitis $\mathrm{C}$ therapeutics and the financial burden on a state correctional system. Journal of Urban Health 2015; 92: 635-649.

14. Razavi H, et al. The present and future disease burden of hepatitis $\mathrm{C}$ virus (HCV) infection with today's treatment paradigm. Journal of Viral Hepatitis 2014; 21: 34-59.

15. Centers for Disease and Control Prevention (CDC). Hepatitis surveillance. Atlanta, GA: CDC, 2006.

16. Daniels D, et al. Surveillance for acute viral hepatitis United States, 2007. Morbidity and Mortality Weekly Reports. Surveillance Summaries 2009; 58: 1-27.

17. Wasley A, et al. Surveillance for acute viral hepatitis United States, 2005. Morbidity and Mortality Weekly Reports. Surveillance Summaries 2007; 56: 1-24.

18. Wasley A, et al. Surveillance for acute viral hepatitis United States, 2006. Morbidity and Mortality Weekly Reports. Surveillance Summaries 2008; 57: 1-24.

19. Malling M. Acute and chronic hepatitis C 2009: Statens Serum Institut, 2010. Report No. 19.

20. Thein HH, et al. Estimation of stage-specific fibrosis progression rates in chronic hepatitis $\mathrm{C}$ virus infection: A meta-analysis and meta-regression. Hepatology 2008; 48: 418-431.

21. Siebert U, Sroczynski G. Effectiveness and costeffectiveness of initial combination therapy with interferon/peginterferon plus ribavirin in patients with chronic hepatitis $\mathrm{C}$ in germany: A health technology assessment commissioned by the german federal ministry of health and social security. International Journal of Technology Assessment in Health Care 2005; 21: 55-65.

22. Sennfält K, et al. Cost-effectiveness of interferon alfa-2b with and without ribavirin as therapy for chronic hepatitis $\mathrm{C}$ in sweden. Scandinavian Journal of Gastroenterology 2001; 36: 870-876.

23. Freeman AJ, et al. Estimating progression to cirrhosis in chronic hepatitis C virus infection. Hepatology 2001; 34: 809-816.

24. Fu B, Wang W, Shi X. Impact of delayed diagnosis time in estimating progression rates to hepatitis C virusrelated cirrhosis and death. Statistical Methods in Medical Research 2015; 24: 693-710.

25. Micallef JM, Kaldor JM, Dore GJ. Spontaneous viral clearance following acute hepatitis $\mathrm{C}$ infection: A systematic review of longitudinal studies. Journal of Viral Hepatitis 2006; 13: 34-41.

26. Centers for Disease and Control Prevention. Historical reported cases and estimates. Atlanta, GA: Division of Viral Hepatitis and National Center for HIV/AIDS, Viral Hepatitis, STD, and TB Prevention, 2015.

27. Centers for Disease and Control Prevention (CDC). Viral hepatitis surveillance: United States, 2011. Division of Viral Hepatitis, Atlanta, GA: National Center for HIV/ AIDS, Viral Hepatitis, STD, and TB Prevention, 2011.

28. Church D. Hepatitis C surveillance: What does the actual epidemic look like? Commonwealth of Massachusetts Department of Public Health, 2014.

29. Division of Viral Hepatitis, Centers for Disease Control and Prevention (CDC). Viral hepatitis statistics and surveillance. Atlanta, GA: CDC, 2015. 
30. Onofrey S, et al. Underascertainment of acute hepatitis $\mathrm{C}$ virus infections in the U.S. Surveillance system: a case series and chart review. Annals of Internal Medicine 2015; 163: 254-261.

31. Edlin BR, et al. Toward a more accurate estimate of the prevalence of hepatitis $\mathrm{C}$ in the United States. Hepatology 2015; 62: 1353-1363.

32. Holmberg SD, et al. Hepatitis $\mathrm{C}$ in the United States. New England Journal of Medicine 2013; 368: 1859-1861.

33. Institute of Medicine (IOM). Hepatitis and Liver Cancer: a National Strategy for Prevention and Control of Hepatitis $B$ and $C$. Washington, DC: The National Academies Press, 2010.

34. Davis GL, et al. Aging of hepatitis $\mathrm{C}$ virus (HCV)-infected persons in the United States: A multiple cohort model of HCV prevalence and disease progression. Gastroenterology 2010; 138: 513-521.

35. Bruggmann $\mathbf{P}$, et al. Historical epidemiology of hepatitis $\mathrm{C}$ virus (HCV) in selected countries. Journal of Viral Hepatitis 2014; 21: 5-33.

36. Volk ML, et al. Public health impact of antiviral therapy for hepatitis $\mathrm{C}$ in the United States. Hepatology 2009; 50: 1750-1755.

37. Kershenobich D, et al. Applying a system approach to forecast the total hepatitis $\mathrm{C}$ virus-infected population size: Model validation using US data. Liver International 2011; 31: 4-17.

38. Centers for Disease Control and Prevention. Hepatitis C: confirmed antibody, RNA (HCV-RNA), and genotype. National Health and Nutrition Examination Survey, 2015.
39. Van Nuys K, et al. Broad hepatitis C treatment scenarios return substantial health gains, but capacity is a concern. Health Affairs 2015; 34: 1666-1674.

40. Boccato $\mathbf{S}$, et al. Fibrosis progression in initially mild chronic hepatitis C. Journal of Viral Hepatitis 2006; 13: 297-302.

41. Zibbell JE, et al. Increases in hepatitis $C$ virus infection related to injection drug use among persons aged $\leqslant 30$ years - Kentucky, Tennessee, Virginia, and West Virginia, 2006-2012. Morbidity and Mortality Weekly Report 2015; 64: 453-458.

42. Anon. SAMSHA, ASAM launch new buprenorphine mentoring program. Alcoholism \& Drug Abuse Weekly 2004; 16: 1-3.

43. Mahajan R, et al. Mortality among persons in care with hepatitis $\mathrm{C}$ virus infection: The chronic hepatitis cohort study (CHeCS), 2006-2010. Clinical Infectious Diseases 2014; 58: 1055-1061.

44. Sacks-Davis R, et al. Hepatitis C virus reinfection and spontaneous clearance of reinfection - the inc3 study. Journal of Infectious Diseases 2015; 212: 1407-1419.

45. Center for Health Law and Policy Innovation of Harvard Law School. Examining hepatitis $\mathrm{C}$ treatment access: a review of select state medicaid fee-for-service and managed care program - Rhode Island, 2015.

46. Müllhaupt B, et al. Modeling the health and economic burden of hepatitis $\mathrm{C}$ virus in switzerland. PLOS ONE 2015; 10: e0125214.

47. Chahal HS, et al. Cost-effectiveness of early treatment of hepatitis $\mathrm{C}$ virus genotype 1 by stage of liver fibrosis in a US treatment-naive population. JAMA Internal Medicine 2016; 176: 65-73. 